\title{
Predictive and holistic energy distribution for hybrid electric vehicles
}

\author{
Zlatina Dimitrova ${ }^{1,2, *}$ \\ ${ }^{1}$ PSA Groupe, Research and Innovation Department, 78943 Vélizy- Villacoublay, France \\ ${ }^{2}$ Ecole Polytechnique Fédérale de Lausanne, IPESE, 1100 Lausanne, Switzerland
}

\begin{abstract}
The improvement of the efficiency of vehicle energy systems stimulate active search to find innovative solutions during the design process. Engineers can use computer-aided processes to find automatically the best design solutions. This kind of approach named "multi-objective optimization" is based on genetic algorithms. The idea is to obtain simultaneously a population of possible design solutions corresponding to the most efficient energy system definition for a vehicle. These solutions will be optimal from technical and economic point of view. This paper presents a systematic optimization methodology for vehicle energy systems that delivers the designs of optimal vehicle powertrain solutions and their optimal operating strategies in a holistic way. The methodology is applied on D class hybrid electric vehicles, in order to define the powertrain configurations, to estimate the cost of the powertrain equipment and the optimal operating parameters. The optimal designs and operating strategies are researched for the normalized European driving cycle. The optimization can be done on-line and consider the next step of the driving cycle, which allow for predictive energy distribution strategies.
\end{abstract}

\section{Introduction}

\subsection{Energy management strategies}

Energy management strategies studies are done most of the time on the local level so that the energy consumption and performance of vehicles are improved. Zhang et al. did in [1] an analysis of the energy management strategies for hybrid electric vehicles: driving cycle recognition/prediction algorithms, integrated multiobjective, coordinated optimization energy management strategies. Most of the strategies are heuristics based and Trovao et Antunes proposed in [2] an analysis of metaheuristic methods for power management of a dual energy storage system for electric vehicles. Chen and al. proposed in [3] an energy management strategy for a series of plug-in hybrid electric vehicle. They applied a number of quadratic equations to determine the engine fuel-rate with respect to battery power. The dynamic programming used for the solution of the optimal control problems is illustrated in [4], where $\mathrm{Xu}$ et al. study a multi-objective component sizing based on optimal energy management strategy of fuel cell electric vehicles. On-line predictive energy management is studied in [5].

This paper proposes a method for energy management of hybrid electric vehicles based on a holistic controller. A continuous energy distribution function is proposed. The main idea is to optimize in a simultaneous way the continuous energy distribution function. The optimization is done with a genetic algorithm. The optimization can be done on-line and considers the next step of the driving cycle, which is allowing predictive energy distribution strategies. The optimal sizing of the powertrain based on techno economic criteria is defined.

\section{Methodology}

\subsection{Energy distribution function}

The vehicle simulation tool is SIMULINK $®$. The vehicle model is based on mechanical and electrical flows. The level of the model is quasi-static. The vehicle is able to follow dynamic profiles generated from a library of driving cycles. The model has a loop energy management structure, linked to the required mechanical power, to follow the dynamic cycle. This energy management loop is called "back and forward" and allows, for a given design of the vehicle powertrain, to simulate the energy consumption of the vehicle, on the given driving profile. The energy flow is computed backwards from the wheels to the energy sources. The model is introduced and explained in details by the author in [6] (Fig. 1).

Hybrid- electric vehicles differ also according to the degree of hybridization of the powertrain and the battery capacity. In the optimization part the battery capacity and the hybridation ratio are part of the decision variables.

* Corresponding author: zlatina.dimitrova@mpsa.com 


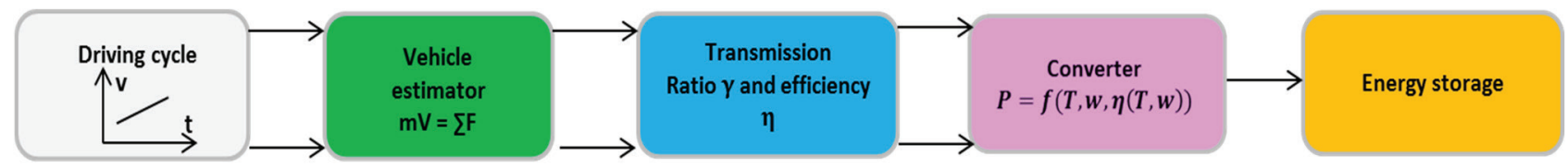

Fig. 1. Quasi-static model of the parallel thermal electric hybrid [6].

The solutions are classified according to the functional classification - mild, hybrid electric, plug-in hybrid electric and purely electric vehicles.

A power distribution strategy is put into place at the coupling of the electric motor and the combustion engine torques. This strategy defines the hybrid ratio, which represents the power contribution of the electric side of the powertrain. The model is based on a commercial D class diesel hybrid electric vehicle, with parallel hybrid architecture.

The degree of hybridization is defined in (1), as the ratio between the electric power and the total power. All symbols in the equations are explained in the nomenclature. The benefits, i.e. the reduction of the energy consumption and the additional cost associated with the hybridization, increase with the degree of hybridization. There are various possibilities to develop energy optimization strategies and most of them are heuristic based. Their guiding principles are that in the hybrid vehicle the engine should be used when its efficiency is relatively high and the battery charge and discharge should be regulated so that the state of charge remains within predefined limits. The operating modes of the powertrain are mapped, for example, the torque request is expressed as a function of the vehicle speed demand and the state of the charge of the battery [7].

$$
H_{r}=\frac{P_{E M}}{P_{T O T}}=\frac{P_{E M, \max }}{P_{E M, \max }+P_{I C E, \max }} .
$$

In this study, instead of having a discontinuous map with modes switching, a continuous function, relating to the hybridization ratio (the electric motor contribution), the state of charge of the battery and the speed demand is developed. Equation (2) gives the generic expression.

$$
H_{r}=f(V, S o C) \text {. }
$$

Thus, the parameters relating to the state variables of the electric motor usage can be optimized for minimal energy consumption and minimal size of the powertrain components. In the multi-objective optimization problem these are the variables for operating strategies. The optimization problem is solved with an evolutionary genetic algorithm and the optimal operating strategies solution is solved in a holistic way. This is one of the novelties of this work. The batteries have low specific energy in comparison to fossil fuels. The energy management strategy makes use of the electric drive at low speeds and high state of charge ( $\mathrm{SoC}$ ) of the battery. The hybridization ratio decreases at high speeds and low $\mathrm{SoC}$ in order to save the battery from overload. A continuous S-curve function is suitable to archive these requirements (3).

$$
H_{r}(S o C)=3+\left(\frac{c_{1} \times S o C-\left(S o C_{\min }+S o C_{\max }\right)}{S o C_{\min }+S o C_{\max }}\right)^{3} .
$$

The behaviour of such shape is illustrated in Fig. 2, where $S o C_{\text {min }}=0,3$ and $S o C_{\text {max }}=0,8$.
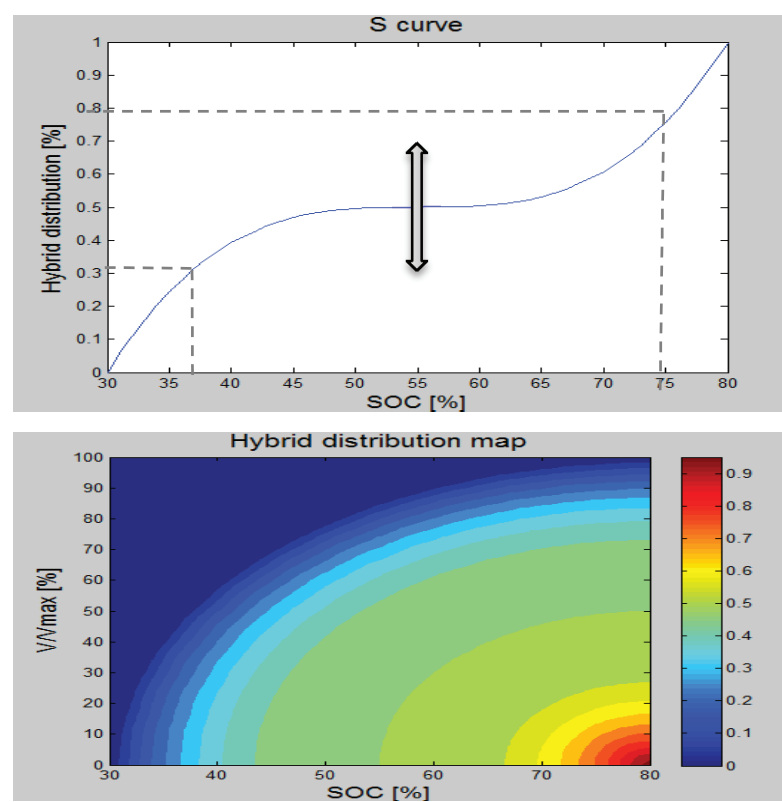

Fig 2. Hybridization ratio strategy.

During simulation, if the $S o C$ is high, the energy management computes a high hybridization ratio and drains the battery, so its $S o C$ will tend to the central plateau of the $S$ - curve. On other hand, if the $S o C$ is below the plateau, the strategy computes a low ratio of hybridization in order for the battery to get recharged during the regenerative braking. The strategy has a stabilizing effect on the state of charge. Coefficients $c_{1}$ and $c_{2}$ are responsible for the shape of the energy distribution. Parameter $c_{1}$ influences the height of the plateau. To extend the strategy to the vehicle speed, the $S$ curve is rotated in space, around the upper vertical axe. The speed axe is normalized using the maximal speed $V_{\max }$ of the driving cycle. Parameter $c_{2}$ is related to the stretch of the rotation. A simplified vehicle objective cost function is constructed (5), taking into account the vehicle powertrain cost (production) (4) and vehicle nominal cost.

$$
\begin{aligned}
\operatorname{Cost}_{\text {powertrain }} & =\operatorname{Cost}_{I C E}+\operatorname{Cost}_{E M}+\text { Cost }_{\text {battery }} \\
\text { Cost }_{\text {vehicle }} & =\text { Cost }_{\text {powertrain }}+\text { Cost }_{\text {car_shell }} .
\end{aligned}
$$

The multi-objective optimization tool used in this paper is presented in Fig. 3. 


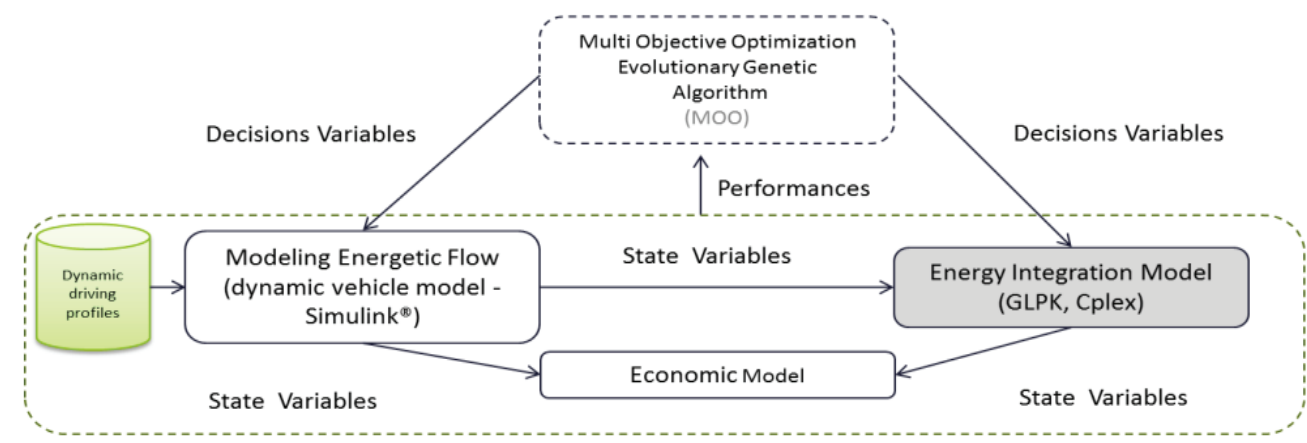

Fig. 3. Architecture of the multi-objective optimization tool.

The general computational approach has already been described in [6]. The superstructure contains a physical vehicle simulation model, with dynamic and thermal layouts. The cost equations are written in the economic model. The energy integration model uses the results from the dynamic and thermal flows calculations. The optimizer is based on a genetic algorithm.

\subsection{Algorithm for holistic energy distribution}

Fig. 4 presents a predictive energy management system for a hybrid vehicle, where the system uses vehicle input information, such as present location, time, 3-D maps, driving history, etc., to better determine the engine and motor power commands. The predictive energy management system includes a learning and predictions process block.

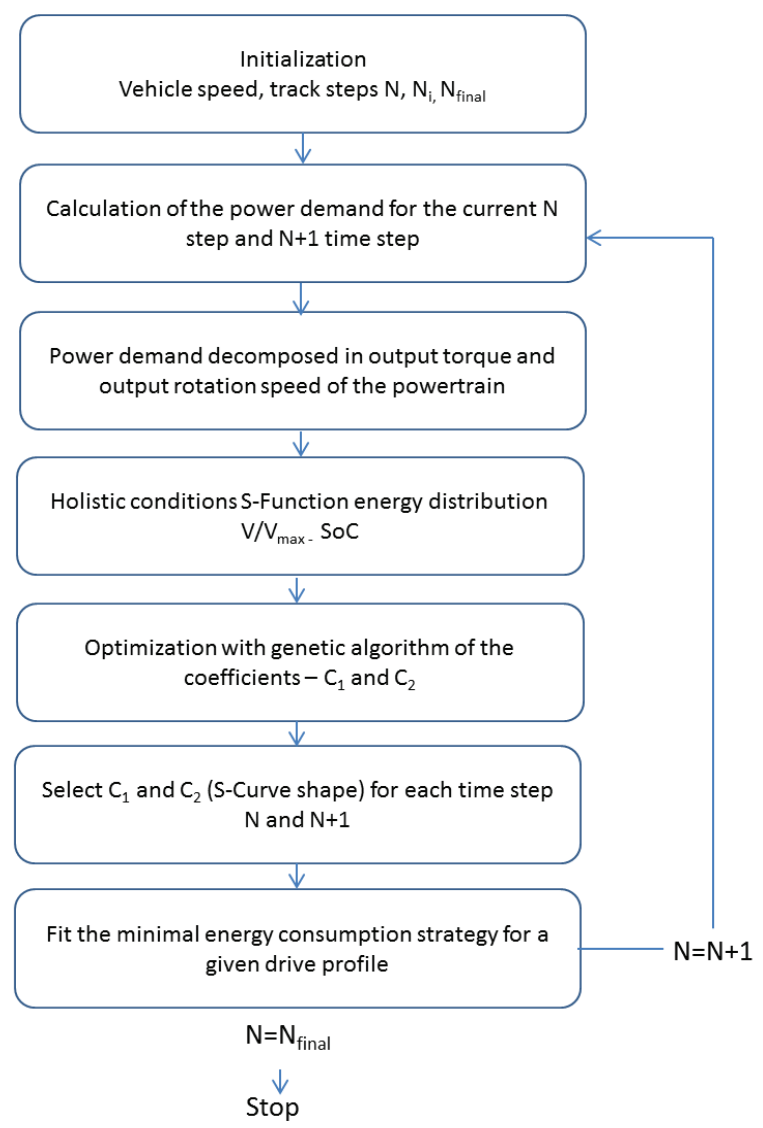

Fig. 4. Algorithm for a holistic energy distribution.
In this stage input information is obtained, a driver power demand for a series of $N$ samples of future time horizon starting from the present is calculated. The calculated driver power demand is then applied to a predictive control strategy process block that generates the engine and motor power commands for each $N$ sample. The predictive control strategy block employs optimization techniques based on genetic algorithms and is based on continuous $S$-curve shaped energy distribution curve to find the optimum sequence of power commands for the series of $N$ samples (Fig. 4).

\section{Results. Application in hybrid electric vehicles}

\subsection{Problem definition}

A hybrid vehicle with multiple propulsion systems can be operated independently or together. The model contents are the electric machine, battery, supercapacitors, thermal engine and fuel tank, with diesel fuel. The vehicle model represents a commercial D- class [8] vehicle with a thermal electric powertrain.

In this study, instead of defining one vehicle with a set of parameters and then studying its performances over various driving cycles, the reverse is done. For each use the powertrain components and the energy management parameters are optimized. Thus, each usage has its optimal vehicle design. The objective is to size the components of the hybrid powertrain. The converters and the storage tanks are defined and the optimal operating strategy is estimated, regarding the energy consumption and the cost objectives. A two objective optimization is considered. The energy consumption and the powertrain cost are minimized. The optimization problem is defined as:

$\min \left(-\eta_{\text {powertrain }}(x), \operatorname{Cost}_{\text {vehicle }}(x)\right)$, s.t. $x \in X_{\text {decision_variables }}(6)$

The decision variables for the powertrain design and energy management are defined in Table 1.

After each iteration of the model, the mean powertrain efficiency in traction is calculated as:

$$
\eta_{\text {powertrain }}=\text { mean }\left(\frac{P_{\text {wheels }}}{P_{\text {fuel }}+P_{\text {battery }}}\right) .
$$


Table 1. Decision variables for design and energy management.

\begin{tabular}{|l|l|}
\hline \multicolumn{1}{|c|}{ Decision variable for design } & \multicolumn{1}{c|}{ Range } \\
\hline ICE displacement volume $[\mathrm{L}]$ & {$[0,8-1-1,4-1,6-2,2]$} \\
\hline Electric motor rated power $[\mathrm{kW}]$ & {$[1-150]$} \\
\hline Battery energy $[\mathrm{kWh}]$ & {$[5-50]$} \\
\hline Energy strategy parameter $c_{1}[-]$ & {$[1,8-2,4]$} \\
\hline Energy strategy parameter $c_{2}[-]$ & {$[0,1-10]$} \\
\hline
\end{tabular}

An additional step is to open the optimization of the parameters of the energy management in a predictive way. The trajectory is known and the best energy management should be used to minimize the fuel consumption of the vehicles during its trajectory. The possibility of the optimization structure for predictive energy management is illustrated by a simple example using the well-known New European Driving Cycle (NEDC). The cycle is divided into specific numbers and length of segments. Then, the multi-objective optimization is applied to maximize the powertrain efficiency. For each segment one can see the optimal energy management function.

\subsection{Results and discussion}

The cycle is divided into segments with a time division of 1 minute as time interval. The algorithm will look what is the trajectory 1 minute ahead and find the best parameters for the hybrid electric vehicle (HEV) (Fig. 5). By taking the value of the parameters of the closest point to the average efficiency and applying the same parameters into a complete NEDC run, one obtains the following results (Table 2). The optimization result is very close in terms of efficiency and cost to the average point, without decomposition of the cycle.

In the second stage a hybrid electric vehicle with 7 $\mathrm{kWh}$ of battery capacity is considered for the study. The powertrain parameters are listed in Table 3.

The optimal set of the S-Curve parameters is researched to minimize the fuel consumption of the vehicle for each cycle segment. Then the vehicles choose the optimal energy distribution as a function of the trajectory. The average value of the consumptions for the segmented NEDC is equal to $4,2 \mathrm{~L} / 100 \mathrm{~km}$.

The best fuel consumption with their associated SCurve parameters, for each segment, are represented in Table 4.

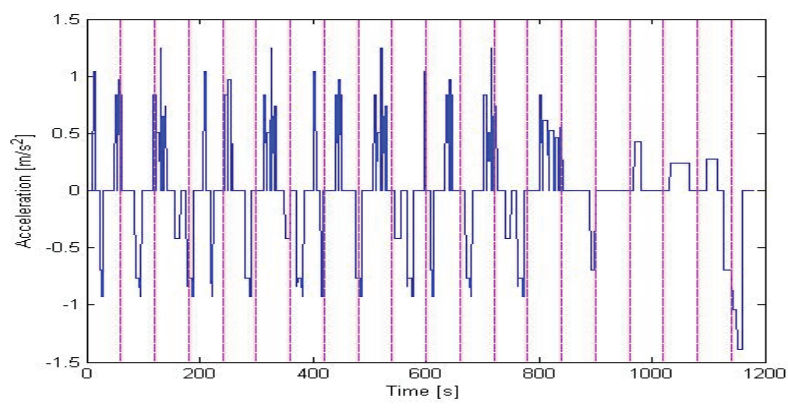

Fig. 5. Segmentation of the NEDC - one minute duration of the segments.
Table 2. Design and energy management parameters on a complete NEDC.

\begin{tabular}{|l|l|}
\hline Parameters & Value \\
\hline Powertrain efficiency [-] & 0,325 \\
\hline Investment cost [€] & 55428 \\
\hline Displacement volume [L] & 1,9 \\
\hline Electric motor rated power [kW] & 113 \\
\hline Battery size [kWh] & 24 \\
\hline Energy strategy parameter 1 & 1,8 \\
\hline Energy strategy parameter 2 & 0,5 \\
\hline Consumption [L/ 100 km] & 3 \\
\hline Vehicle mass [kg] & 2187 \\
\hline
\end{tabular}

Table 3. Design of the HEV for the NEDC segmentation.

\begin{tabular}{|l|l|}
\hline Parameters & Value \\
\hline ICE displacement volume $[\mathrm{L}]$ & 2,2 \\
\hline Electric motor rated power $[\mathrm{kW}]$ & 40 \\
\hline Battery capacity $[\mathrm{kWh}]$ & 7 \\
\hline
\end{tabular}

Table 4. Optimal fuel consumption on the NEDC segments.

\begin{tabular}{|l|l|l|l|}
\hline ID & $\begin{array}{l}\text { Fuel } \\
\text { consumption } \\
{[\mathbf{L} / \mathbf{1 0 0} \mathbf{~ k m}]}\end{array}$ & $\begin{array}{l}\text { Energy } \\
\text { distribution } \\
\boldsymbol{c}_{\mathbf{1}} \\
\text { parameter }\end{array}$ & $\begin{array}{l}\text { Energy } \\
\text { distribution } \\
\boldsymbol{c}_{2} \text { parameter }\end{array}$ \\
\hline 1 & 5,5 & 1,80 & 0,49 \\
\hline 2 & 3,5 & 1,84 & 0,46 \\
\hline 3 & 4,3 & 2,02 & 0,27 \\
\hline 4 & 2,4 & 2,00 & 0,39 \\
\hline 5 & 3,9 & 1,89 & 0,20 \\
\hline 6 & 5,5 & 2,16 & 0,38 \\
\hline 7 & 2,0 & 2,02 & 0,17 \\
\hline 8 & 4,6 & 1,80 & 0,33 \\
\hline 9 & 6,1 & 1.93 & 0,40 \\
\hline 10 & 1,5 & 1,80 & 0,41 \\
\hline 11 & 7,9 & 2,37 & 0,18 \\
\hline 12 & 3,3 & 1,80 & 0,51 \\
\hline 13 & 4,0 & 2,30 & 0,14 \\
\hline 14 & 6,6 & 2,15 & 0,15 \\
\hline 15 & 4,2 & 2,21 & 0,36 \\
\hline 16 & 2,7 & 1,80 & 0,44 \\
\hline 17 & 4,2 & 1,87 & 0,31 \\
\hline 18 & 4,9 & 2,14 & 0,38 \\
\hline 19 & 5,9 & 2,20 & 0,34 \\
\hline 20 & 1,6 & 2,22 & 0,51 \\
\hline & & & \\
\hline
\end{tabular}

As illustrated in Table 4, segment 10 demonstrates the lowest fuel consumption and segment 11 demonstrates the highest fuel consumption. The difference in the fuel consumption between these two segments can be explained by the different hybridation ratio that the holistic energy management controller defines. The energy distribution function for segment 10 shows in Fig. $6 \mathrm{a}$, a dominant level of the electric traction that is used. This is the reason for the lowest fuel consumption results. The energy distribution function for segment 11 is shown in Fig. 6b, a dominant thermal engine mode. Thus, the highest fuel consumption is estimated. 


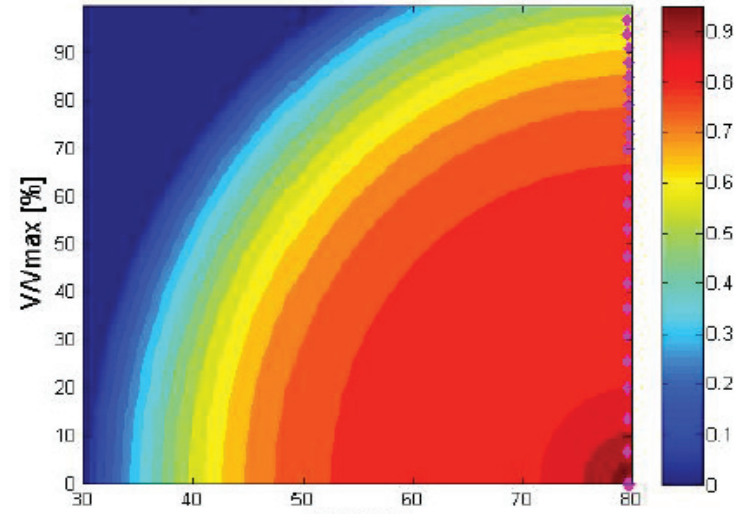

a)

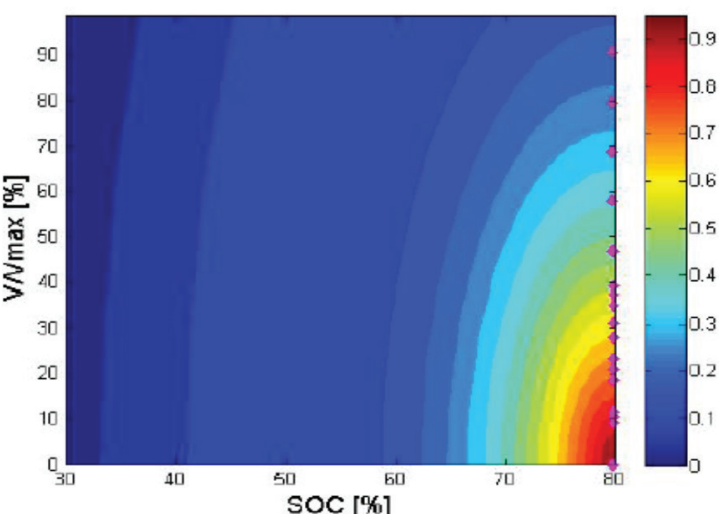

b)

Fig. 6. $S$-Curve energy distribution function a) dominant electric motor mode b) dominant thermal engine mode.

\section{Conclusions}

This paper proposes a holistic method for control and design optimization of hybrid electric vehicles. It is based on a multi-objective optimization using a genetic algorithm. The optimal size of the propulsion system is based on an optimal energy function definition. A continuous energy distribution function is proposed for that. The main idea there is to optimize in a simultaneous way the continuous energy distribution function. The optimization can be done on-line and consider the next step of the driving cycle, which is allowing predictive energy distribution strategies. The optimal designs are given for every segment of the NEDC and they are compared on the average optimal design. The fuel consumption results are explained, related to the energy distribution function, applied for the segments. The method could be used for predictive control and for cost effective design, which will cover the power demands for the real drives.

\section{Nomenclature}

$c_{1}, c_{2}$,- energy management function coefficients in [-]

$\mathrm{EM}$ - electric motor

$\mathrm{F}-$ force in $[\mathrm{N}]$

GLPK, Cplex - solvers

HEV- Hybrid Electric Vehicle

$H_{r}$ - hybridization ratio in [-]

ICE - internal combustion engine

$\mathrm{m}$ - vehicle mass in [kg]

MOO - Multi Objective Optimization

NEDC - New European Driving cycle

$P$ - power of the drive shaft in [kW]

$P_{\text {battery }}$ - power delivered from the battery in [kW]

$P_{E M}$ - power of the electric motor in [kW]

$P_{\text {fuel }}$ - power in the fuel in $[\mathrm{kW}]$

$P_{I C E}$ - power of the internal combustion engine in [kW]

$P_{T O T}$ - total power of the vehicle powertrain in $[\mathrm{kW}]$

$P_{\text {wheels }}$ - power transmitted to the wheels in $[\mathrm{kW}]$

$T-$ torque on the drive shaft in [Nm]

SoC - state of charge of the battery in [\%]

$\mathrm{V}$ - vehicle speed in $[\mathrm{m} / \mathrm{s}]$

$w$ - rotation speed [rpm]

$\gamma$ - gear ratio [-]

$\eta_{\text {powertrain }}$ - powertrain efficiency in [-]

\section{References}

1. P. Zhang, F. Yan, C. Du, Renewable \& Sustainable Energy Reviews, 48, 88-104, (2015)

2. J. Trovão, C. Antunes, Energy Conversion and Management, 95, 281-296, (2015)

3. Y. Xu, D. Liu, Q. Wei, Energy Conversion and Management, 103, 553-561, (2015)

4. Z. Chen, B. Xia, C.You, C. Mi, Applied Energy, 145, 172-179, (2015)

5. L. Li, S. You, C. Yang, B. Yan, J. Song, Z. Chen, Applied Energy, 162, 868-879, (2016)

6. Dimitrova, F. Maréchal, Energy, 91, 630-644, (2015)

7. L. Guzzella. Vehicle propulsion systems, (2013)

8. La centrale, fiche technique de la Peugeot $508 \mathrm{SW}$, http://www.lacentrale.fr [accessed 17.06.17]. 